Lindegren, C. C., Lindegren, G. \& Desborough S. (1959). J. gen. Microbiol. 20, 504-509

\title{
Gene Controlled Resistance and Sensitivity to Caffeine and Nicotine in Saccharomyces
}

\author{
By C. C. LINDEGREN, GERTRUDE LINDEGREN AND \\ SHARON DESBOROUGH \\ Biological Research Laboratory, Southern Illinois University, Carbondale, \\ Illinois, U.S.A.
}

SUMMARY: A significant extension in the variety of gene-markers available in Saccharomyces has been achieved by exploiting resistance and sensitivity to a variety of poisons. Although resistance to many poisons was obviously heritable, genecontrol could not be demonstrated, presumably because other genes modified the phenotypes. Gene-control of resistance and sensitivity for both nicotine and caffeine was demonstrated by tetrad analysis and by the observation of regular Mendelian segregation of the characters among the progeny of closely related hybrids.

The project of constructing maps in Saccharomyces began with the exploitation of gene-markers controlling fermentation of various carbohydrates and was extended to include nutrilite requirements for various B-vitamins, amino acids and nucleic acid components. In this programme it was planned to use nutrilite requirements which could be diagnosed in any combination in the progeny of hybrids, but only a limited number of these are available, because substitution is relatively common. B-vitamin requirements have the disadvantage that the vitamin must be used in very low concentrations. About twenty suitable gene markers have been discovered in these categories and incorporated into the breeding stock. The search for new genetic markers has led to considering the possibility of using resistance and sensitivity to various poisons, since resistance and sensitivity to specific poisons seems to be a highly specific characteristic, and may make available a larger number of genemarkers than the nutritional or fermentative markers.

\section{METHODS}

Three procedures for diagnosing resistance and sensitivity were used.

(1) The test-tube method in which the organisms are inoculated into a tube containing a defined (or a complete) nutrient medium to which a specified amount of the toxic agent has been added (Middlekauff et al. 1957). In this procedure, a numerical value for the amount of growth can be obtained by readings made by optical density methods, but repeated tests do not always give the same results because of mutation and selection.

(2) The disk method in which paper disks (saturated with solutions containing different concentrations of the toxic agent) are placed on an agar surface freshly inoculated with the organisms to be tested is used with poisons that are not readily soluble or which precipitate or colour the medium. The tube method 
is limited to a critical low concentration of poison which will permit growth to begin (because all the organisms in the tube are exposed equally to the toxic agent); but with the disk method it is possible to use disks saturated with concentrations of poison from $\mathbf{5}$ to $\mathbf{5 0}$ times stronger than could be used in a tube. The disk method enables one to observe the frequency of resistant secondary colonies in the clear zone (Pl. 1). In addition, agar overlay containing triphenyltetrazolium chloride (TTC) can be applied to the colonies to determine whether or not 'respiratory deficiencies' have been produced by the poison. Pink (adenine-requiring) cultures turn white or light brown when they become respiratory deficient, and in the disk method one may detect the occurrence of respiratory deficiency (due to the poison) without the TTC agar overlay. The disk method is used to discriminate between resistance and sensitivity to cadmium, propamidine, actidione and nicotine, and the tube method is used to discriminate between resistance and sensitivity to cadmium, actidione, caffeine and cobalt.

(3) The toxic agent may be incorporated into the agar and this method is used in distinguishing between resistance and sensitivity to copper. Copper is added to a full complex medium and the plate method permits the observation of secondary colonies (Ashida \& Imai, 1956; Brenes-Pomales, Lindegren \& Lindegren, 1955).

\section{Diagnostic criteria of resistance to poisons}

A few of the problems involved in diagnosing the genotypes of resistance and sensitivity must be considered. Cultures in the various pedigrees to be examined must be maintained on a complete complex (natural) nutrient medium which does not contain any known toxic agent. Cultures must not be recovered and placed in stock after the exposure to the poison, since exposure to toxic agents might lead to the overgrowth of the sensitive culture by resistant forms and to the development of double mutants in the resistant culture.

The diagnostic concentration must be one that kills all the sensitive organisms and permits all the resistant ones to survive and grow. Too high a concentration will kill both resistant and sensitive organisms and too low will permit resistant and sensitive organisms to grow and thus confuse the genetical analysis. It is interesting that more of the cultures survived and grew

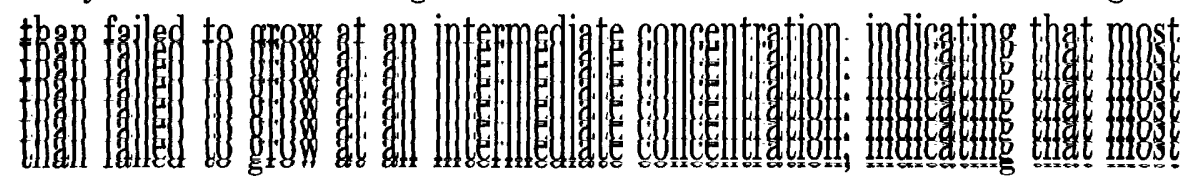

organisms used were able to adapt to moderate concentrations of poisons.

Sibling cultures usually showed significant differences in sensitivity and resistance when grown in intermediate concentrations of poison, and replicate tests of resistance and sensitivity were usually reproducible, indicating that resistance and sensitivity were heritable traits.

With most poisons (propamidine, propamidine isethionate, mycostatin, salicylanilide, undecylenic acid, $p$-nitrophenol, 8-hydroxyquinoline, juglone, mercuric chloride and silver nitrate) regular monohybrid segregations were not discovered despite the fact that the characters were obviously inheritable, as inferred from the reproducibility of the tests for resistance and sensitivity. One 
would only expect regular monohybrid ratios if the modifiers of the genes of resistance and sensitivity produced only trivial effects on the phenotype and if the mutation rate were relatively low. It was surprising, therefore, to discover several instances of monohybrid segregation of resistance and sensitivity for several different toxic agents in unselected pedigrees.

Failures to replicate the diagnosis were fewer than might have been expected, and were usually interpreted to indicate that the selection of a resistant variant had occurred (Middlekauff et al. 1956).

\section{Secondary colonies}

Resistant cultures grew right up to the edge of the disk, but sensitive cultures left a clear zone around the disk. The clear zones around the disks of some sensitive cultures contained secondary resistant colonies. Sensitive cultures could be differentiated into those in which resistant variants occurred and those in which resistant variants did not occur. The frequency of secondary colonies depended on the toxic agent. Secondary colonies were common among copper-sensitive, propamidine-sensitive and cadmium-sensitive cultures, but this may be characteristic of the specific genes or genomes which have been studied. The first actidione-sensitive cultures showed large numbers of secondaries, but the more recent double-mutant controlling actidioneresistance produced fewer secondaries.

A sensitive culture which produces secondary colonies in the clear zone of a plate with the disk method would be expected to produce delayed secondary growth in the test tube and thus be incorrectly diagnosed as a resistant. This problem was analysed in detail in the study of resistance and sensitivity to cadmium (Middlekauff et al. 1956). Resistant cultures which grew rapidly and produced turbidity in 1 or 2 days were easy to distinguish from sensitive cultures which remained clear, i.e. failed to show growth after 10 or more days. Cultures in which growth occurred slowly after about 5 days presented a different problem. Such cultures were assumed to have been originally sensitive and to be distinguished from those which could not grow at all in the presence of the toxic agent, by the possession of an intermediate allele between dominant and the recessive; this was given the symbol of the sensitive allele with

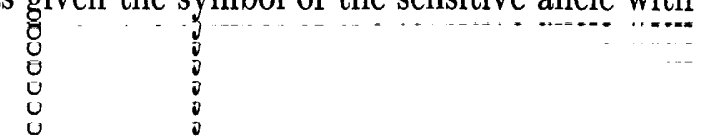

the superscript $M$. This designation is arbitrary since a sensitive culture with a very low mutation rate would be classified as exhibiting the recessive phenotype while one with a high mutation rate would be classified as exhibiting the dominant phenotype.

\section{RESULTS}

\section{Inheritance of resistance to caffeine}

Fries \& Kihlman (1948), Demerec, Bertani \& Flint (1951), and Novick \& Szilard (1952) studied the effects of caffeine on the induction of hereditary, presumably gene-controlled, nutrilite requirements in fungi and bacteria and established the limits of caffeine toxicity. Fries \& Kihlman discovered that $0.05 \%(\mathrm{w} / \mathrm{v})$ caffeine was slightly toxic to Ophiostoma and that all conidia were killed at a concentration of $0 \cdot 2 \%$. Novick \& Szilard grew the colon 
bacillus at a concentration of $0 \cdot 015 \%$ caffeine in the chemostat, presumably because this concentration did not inhibit its growth significantly.

The first discovery of saccharomyces cultures differing in resistance and sensitivity to caffeine was made during a survey of haploid cultures isolated from various pedigrees in previous breeding experiments. Many of the tetrads from these pedigrees showed $2: 2$ segregations of resistance to caffeine (using

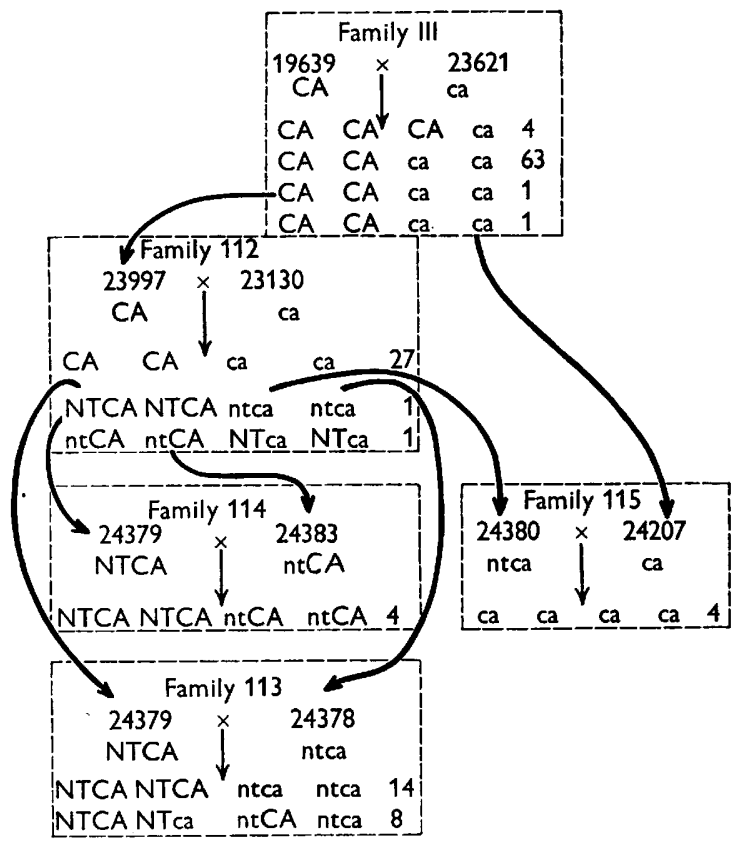

Fig. 1. A pedigree revealing regular Mendelian segregations in tetrads of saccharomyces hybrids heterozygous for genes controlling resistance to sensitivity to caffeine (CA, ca) and/or nicotine (NT, nt).

tubes of diagnostic medium containing $0 \cdot 1 \%$ caffeine). These cultures were used as the source of the pedigrees shown in Fig. 1. Unambiguous segregations were obtained when each haploid culture was tested in two different tubes, one containing 0.2 and one containing $0.4 \%$ caffeine, as compared with the control containing no added caffeine. Many of these tests were confirmed by duplicate tests. Both the 0.2 and $0.4 \%$ caffeine tubes gave comparable results. Mating of sensitive by sensitive, and resistant by resistant, produced progeny in which all offspring resembled the parent culture, thus confirming gene control of resistance to caffeine sensitivity (Fig. 1).

\section{Gene control of resistance to nicotine}

A series of cultures were sampled from Family 111 and indicated that this family was heterozygous for sensitivity and resistance to nicotine and one resistant and one sensitive member of this family were selected as parents for Family 114. The disk method was used in diagnosing resistance to nicotine 
because nicotine both darkens and precipitates the culture medium and cannot, therefore, be used in broth (Fig. 1). Disks were placed in solutions containing $1,3,5$ and $10 \%(\mathrm{w} / \mathrm{v})$ nicotine in water. These disks were then placed on nutrient agar plates previously spread with one of the segregant cultures. Diagnosis of resistance and sensitivity was achieved within $24 \mathrm{hr}$. by inspection of the zone of inhibition. The use of four different concentrations of nicotine minimized ambiguity, since the series showed a progressive difference in size of the clear zone surrounding each disk. Such precipitation as occurred did not interfere with the diagnostic concentration established at the perimeter of diffusion.

\section{The pedigrees}

Family 111 produced sixty-five 2:2 tetrads and four 3:1 tetrads for resistance to caffeine. Family 112 was produced by mating a caffeine-resistant segregant from Family 111 by a caffeine-sensitive segregant from Family 108 and produced 29 tetrads, all of which yielded 2 caffeine-resistant and 2 caffeine-sensitive segregants. A caffeine-sensitive segregant from Family 112 was mated with another caffeine sensitive from Family 111, and produced 4 tetrads (Family 115), all containing only caffeine-sensitive progeny. Family 114 was produced by mating 2 caffeine-resistant clones derived from Family 112 and produced 4 tetrads all of which contained only caffeine-resistant segregants.

Sample cultures of Family 112 (which was heterozygous for genes controlling caffeine-sensitivity) revealed several tetrads containing 2 nicotine-resistant and 2 nicotine-sensitive segregants. One recombination tetrad of the genes controlling caffeine-resistance and nicotine-resistance was discovered. Family 114 was produced by mating a nicotine-resistant by nicotine-sensitive and produced 4 tetrads containing 2 nicotine-sensitive and 2 nicotine-resistant offspring. Family 113 was heterozygous for resistance to both caffeine and nicotine and produced 22 tetrads in which regular segregation for these two genes occurred in every tetrad. Fourteen tetrads carried the genes in the parental combination and 8 tetrads were tetratype.

These data established gene-control of resistance and sensitivity for both caffeine and nicotine. The analysis of tetrads which were heterozygous for other genes has served to locate the new genes on a chromosome carrying eight other markers. This chromosome will be described in detail subsequently (Lindegren et al. 1959).

The writers wish to acknowledge the technical assistance of Mr Bong Kuk Lee. This investigation was supported by a research grant from the Illinois Division of the American Cancer Society.

\section{REFERENCES}

Ashida, J. \& IMAI, M. (1956). Origin of copper resistant cell studies on the adaptation of yeast to copper. XV. Bot. Mag. Tokyo, 69, 560 .

Brenes-Pomales, A., Lindegren, G. \& Lindegren, C. C. (1955). Gene control of copper sensitivity in Saccharomyces. Nature, Lond. 176, 841.

Demerec, M., Bertani, G. \& Fuint, J. (1951). A survey of chemicals for mutagenic action on E. coli. Amer. Nat. 85, 119. 
Journal of General Microbiology, Vol. 20, No. 3

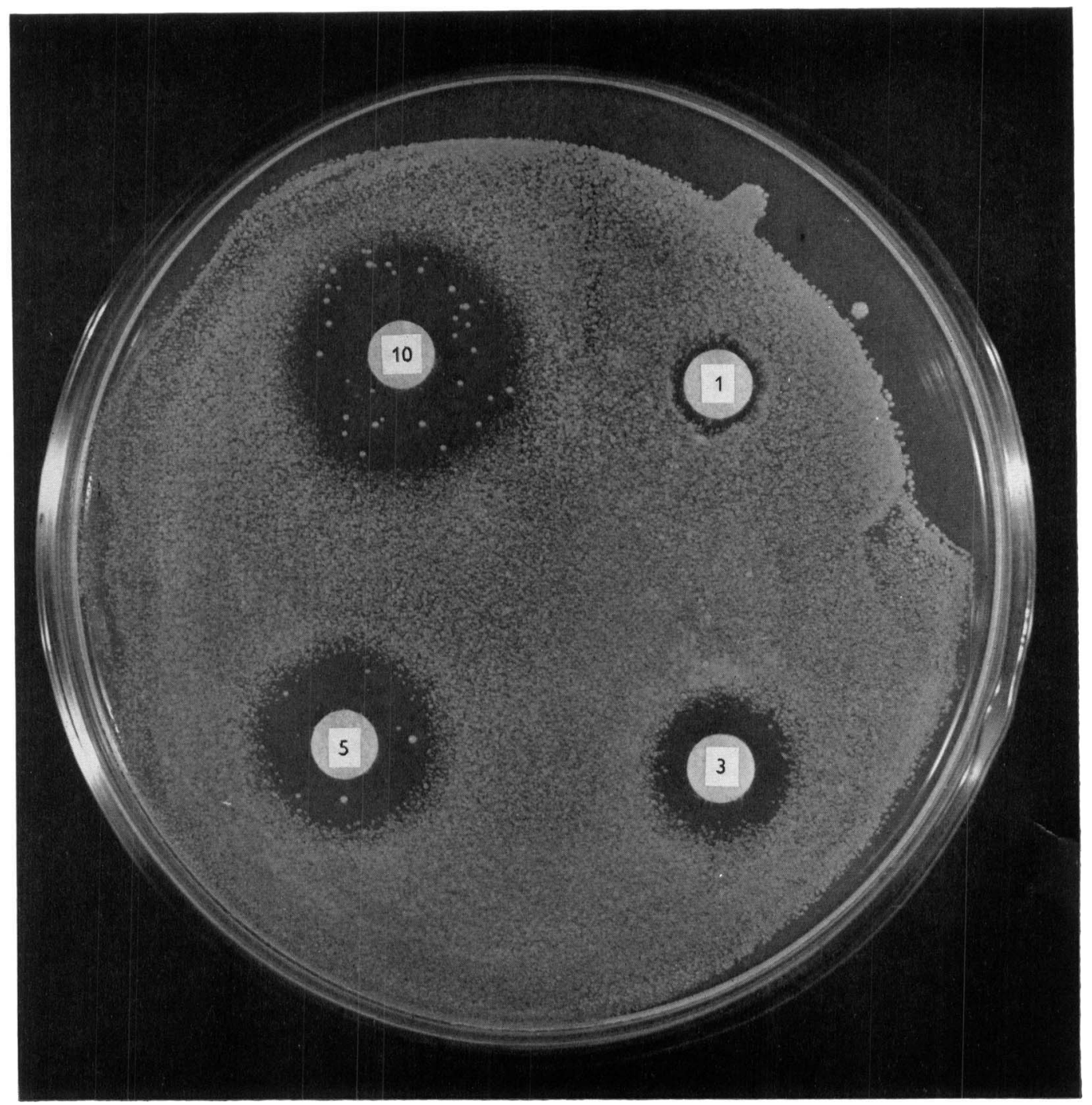

C. C. Lindegren, G. Lindegren \& S. Desborough-New gene-markers in saccharomyces. Plate 1 
Fries, N. \& Kinlman, G. (1948). Fungal mutations obtained with methyl xanthines. Nature, Lond. 162, 573.

Lindegren, C. C., Lindegren, G., Shult, E. \& Desborough, S. (1959). A preliminary report on the chromosome maps of Saccharomyces (in the Press).

Middlekauff, J. E., Hino, S., Yang, Sing-Ping, Lindegren, G. \& Lindegren, C. C. (1956). Gene control of resistance vs. sensitivity to cadmium in Saccharomyces. J. Bact. 72, 796.

Middlekauff, J. E., Hino, S., Yang, Sing-Ping, Lindegren, G. \& Lindegren, C. C. (1957). Gene control of resistance vs. sensitivity to actidione in Saccharomyces. Genetics, $42,66$.

Novick, A. \& Szilard, L. (1952). Anti-mutagens. Nature, Lond. 170, 926.

\section{EXPLANATION OF PLATE}

The effect of placing paper disks saturated with solutions of nicotine on the growth of nicotine-sensitive saccharomyces previously sown on an agar plate. The strength of the different nicotine solutions is indicated on the paper disk. The resistant secondary colonies appearing in the clear zone do not complicate the analysis as they might if the test were performed in a broth tube.

(Received 29 October 1958) 